\title{
Ultrafast all-optical chalcogenide glass photonic circuits
}

\author{
Vahid G. Ta'eed ${ }^{1}$, Neil J. Baker ${ }^{1}$, Libin Fu ${ }^{1}$, Klaus Finsterbusch ${ }^{1}$, Michael R.E. Lamont ${ }^{1}$, \\ David J. Moss ${ }^{1}$, Hong C. Nguyen ${ }^{1}$, Benjamin J. Eggleton ${ }^{1 *}$, Duk Yong Choi ${ }^{2}$, \\ Steven Madden ${ }^{2}$, and Barry Luther-Davies ${ }^{2}$ \\ ${ }^{1}$ Centre for Ultrahigh bandwidth Devices for Optics Systems (CUDOS) \\ School of Physics, University of Sydney, NSW 2006, Australia \\ ${ }^{2}$ Centre for Ultrahigh bandwidth Devices for Optics Systems (CUDOS) \\ Laser Physics Centre, The Australian National University, Canberra, ACT 0200, Australia \\ http://www.cudos.org.au/ \\ *Corresponding author: egg@physics.usyd.edu.au
}

\begin{abstract}
Chalcogenide glasses offer large ultrafast third-order nonlinearities, low two-photon absorption and the absence of free carrier absorption in a photosensitive medium. This unique combination of properties is nearly ideal for all-optical signal processing devices. In this paper we review the key properties of these materials, outline progress in the field and focus on several recent highlights: high quality gratings, signal regeneration, pulse compression and wavelength conversion.
\end{abstract}

(C)2007 Optical Society of America

OCIS codes: (000.0000) General; (190.4370) Nonlinear optics, fibers; (190.4390) Nonlinear optics, integrated optics; (190.7110) Ultrafast nonlinear optics; (230.7390) Waveguides, planar; (060.4370) Nonlinear optics, fibers; (060.7140) Ultrafast processes in fibers

\section{References and links}

1. A. R. Hilton, "Optical properties of chalcogenide glass," J. Non-Cryst Solids 2, 28-39 (1970).

2. J. S. Sanghera, L. B. Shaw, L. E. Busse, V. Q. Nguyen, P. C. Pureza, B. C. Cole, B. B. Harbison, I. D. Aggarwal, R. Mossadegh, F. Kung, D. Talley, D. Roselle, and R. Miklos, "Development and infrared applications of chalcogenide glass optical fibers," Fiber Integr. Opt. 19, 251-274 (2000).

3. J. S. Sanghera, L. B. Shaw, and I. D. Aggarwal, "Applications of chalcogenide glass optical fibers," Comptes Rendus Chimie 5, 873-883 (2002).

4. M. Asobe, H. Itoh, T. Miyazawa, and T. Kanamori, "Efficient and Ultrafast All-Optical Switching Using High Delta-N, Small Core Chalcogenide Glass-Fiber," Electron. Lett. 29, 1966-1968 (1993).

5. L. B. Fu, M. Rochette, V. G. Ta'eed, D. J. Moss, and B. J. Eggleton, "Investigation of self-phase modulation based optical regeneration in single mode As2Se3 chalcogenide glass fiber," Opt. Express 13, 7637-7644 (2005).

6. V. G. Ta'eed, M. Shokooh-Saremi, L. B. Fu, I. C. M. Littler, D. J. Moss, M. Rochette, B. J. Eggleton, Y. L. Ruan, and B. Luther-Davies, "Self-phase modulation-based integrated optical regeneration in chalcogenide waveguides," IEEE J. Sel. Top. Quantum Electron. 12, 360-370 (2006).

7. V. G. Ta'eed, M. Shokooh-Saremi, L. B. Fu, D. J. Moss, M. Rochette, I. C. M. Littler, B. J. Eggleton, Y. L. Ruan, and B. Luther-Davies, "Integrated all-optical pulse regenerator in chalcogenide waveguides," Opt. Lett. 30, 2900-2902 (2005).

8. V. G. Ta'eed, M. R. E. Lamont, D. J. Moss, B. J. Eggleton, D. Y. Choi, S. Madden, and B. Luther-Davies, "All optical wavelength conversion via cross phase modulation in chalcogenide glass rib waveguides," Opt. Express 14, 11242-11247 (2006).

9. V. G. Ta'eed, L. B. Fu, M. Pelusi, M. Rochette, I. C. M. Littler, D. J. Moss, and B. J. Eggleton, "Error free all optical wavelength conversion in highly nonlinear As-Se chalcogenide glass fiber," Opt. Express 14, 10371-10376 (2006).

10. R. E. Slusher, G. Lenz, J. Hodelin, J. Sanghera, L. B. Shaw, and I. D. Aggarwal, "Large Raman gain and nonlinear phase shifts in high-purity As2Se3 chalcogenide fibers," J. Opt. Soc. Am. B 21, 1146-1155 (2004).

11. K. S. Abedin, "Single-frequency Brillouin lasing using singlemode As2Se3 chalcogenide fiber," Opt. Express 14, 4037-4042 (2006).

12. L. B. Fu, A. Fuerbach, I. C. M. Littler, and B. J. Eggleton, "Efficient optical pulse compression using chalcogenide single-mode fibers," Appl. Phys. Lett. 88, 081116 (2006).

13. K. Y. Song, K. S. Abedin, K. Hotate, M. G. Herraez, and L. Thevenaz, "Highly efficient Brillouin slow and fast light using As2Se3 chalcogenide fiber," Opt. Express 14, 5860-5865 (2006). 
14. A. Zakery, and S. R. Elliott, "Optical properties and applications of chalcogenide glasses: a review," J. Non-Cryst Solids 330, 1-12 (2003).

15. K. O. Hill, B. Malo, F. Bilodeau, and D. C. Johnson, "Photosensitivity in Optical Fibers," Annual Review of Materials Science 23, 125-157 (1993).

16. I. D. Aggarwal, and J. S. Sanghera, "Development and applications of chalcogenide glass optical fibers at NRL," J. Optoelectron. Adv. M. 4, 665-678 (2002).

17. L. Brilland, F. Smektala, G. Renversez, T. Chartier, J. Troles, T. N. Nguyen, N. Traynor, and A. Monteville, "Fabrication of complex structures of Holey Fibers in chalcogenide glass," Optics Express 14, 1280-1285 (2006).

18. T. M. Monro, Y. D. West, D. W. Hewak, N. G. R. Broderick, and D. J. Richardson, "Chalcogenide holey fibres," Electron. Lett. 36, 1998-2000 (2000).

19. Y. L. Ruan, W. T. Li, R. Jarvis, N. Madsen, A. Rode, and B. Luther-Davies, "Fabrication and characterization of low loss rib chalcogenide waveguides made by dry etching," Opt. Express 12, 51405145 (2004).

20. J. A. Savage, "Optical-Properties of Chalcogenide Glasses," J. Non-Cryst Solids 47, 101-116 (1982).

21. A. B. Seddon, "Chalcogenide Glasses - a Review of Their Preparation, Properties and Applications," J. Non-Cryst Solids 184, 44-50 (1995).

22. M. Yamane, and Y. Asahara, Glasses for photonics (University Press, Cambridge, 2000).

23. S. R. Elliott, "A Unified Model for Reversible Photostructural Effects in Chalcogenide Glasses," J. NonCryst Solids 81, 71-98 (1986).

24. P. Klocek, ed. Handbook of infrared optical materials (Marcel Dekker, New York, 1991).

25. G. P. Agrawal, Nonlinear Fiber Optics (Academic Press, San Diego, 2001).

26. A. C. Turner, C. Manolatou, B. S. Schmidt, M. Lipson, M. A. Foster, J. E. Sharping, and A. L. Gaeta, "Tailored anomalous group-velocity dispersion in silicon channel waveguides," Opt. Express 14, 4357$4362(2006)$.

27. S. M. Song, and S. Y. Choi, "Spin-coated Ge-Se-Te-Si3N4-CdS chalconitride thin film," J. Non-Cryst Solids 291, 50-55 (2001).

28. T. Kohoutek, T. Wagner, M. Vlcek, M. Vlcek, and M. Frumar, "Spin-coated $\mathrm{As}_{33} \mathrm{~S}_{67-\mathrm{x}} \mathrm{Se}_{\mathrm{x}}$ thin films: the effect of annealing on structure and optical properties," J. Non-Cryst Solids 352, 1563-1566 (2006).

29. T. Wagner, T. Kohoutek, M. Vlcek, M. Vlcek, M. Munzar, and M. Frumar, "Spin-coated Agx(As0.33S0.67)(100-x) films: preparation and structure," J. Non-Cryst Solids 326, 165-169 (2003).

30. G. C. Chern, and I. Lauks, "Spin-Coated Amorphous-Chalcogenide Films," J. Appl. Phys. 53, 6979-6982 (1982).

31. J. M. Gonzalez-Leal, R. Prieto-Alcon, M. Stuchlik, M. Vlcek, S. R. Elliott, and E. Marquez, "Determination of the surface roughness and refractive index of amorphous As40S60 films deposited by spin coating," Opt. Mater. 27, 147-154 (2004).

32. A. K. Mairaj, R. J. Curry, and D. W. Hewak, "Chalcogenide glass thin films through inverted deposition and high velocity spinning," Electron. Lett. 40, 421-422 (2004).

33. K. E. Youden, T. Grevatt, R. W. Eason, H. N. Rutt, R. S. Deol, and G. Wylangowski, "Pulsed-Laser Deposition of Ga-La-S Chalcogenide Glass Thin-Film Optical Wave-Guides," Appl. Phys. Lett. 63, 16011603 (1993).

34. A. V. Rode, A. Zakery, M. Samoc, R. B. Charters, E. G. Gamaly, and B. Luther-Davies, "Laser-deposited As2S3 chalcogenide films for waveguide applications," Appl. Surf. Sci. 197, 481-485 (2002).

35. A. Zoubir, M. Richardson, C. Rivero, A. Schulte, C. Lopez, K. Richardson, N. Ho, and R. Vallee, "Direct femtosecond laser writing of waveguides in As2S3 thin films," Opt. Lett. 29, 748-750 (2004).

36. T. V. Galstyan, J. F. Viens, A. Villeneuve, K. Richardson, and M. A. Duguay, "Photoinduced selfdeveloping relief gratings in thin film chalcogenide $\mathrm{As}_{2} \mathrm{~S}_{3}$ glasses," Journal of Lightwave Technology 15, 1343-1347 (1997).

37. A. Saliminia, A. Villeneuve, T. V. Galstyan, S. LaRochelle, and K. Richardson, "First- and second-order Bragg gratings in single-mode planar waveguides of chalcogenide glasses," Journal of Lightwave Technology 17, 837-842 (1999).

38. K. Tanaka, N. Toyosawa, and H. Hisakuni, "Photoinduced Bragg Gratings in As2s3 Optical Fibers," Opt. Lett. 20, 1976-1978 (1995).

39. R. Vallee, S. Frederick, K. Asatryan, M. Fischer, and T. Galstian, "Real-time observation of Bragg grating formation in As2S3 chalcogenide ridge waveguides," Opt. Commun. 230, 301-307 (2004).

40. N. Ponnampalam, R. G. DeCorby, H. T. Nguyen, P. K. Dwivedi, C. J. Haugen, J. N. McMullin, and S. O. Kasap, "Small core rib waveguides with embedded gratings in As2Se3 glass," Opt. Express 12, 6270-6277 (2004).

41. M. Asobe, T. Ohara, I. Yokohama, and T. Kaino, "Fabrication of Bragg grating in chalcogenide glass fibre using the transverse holographic method," Electron. Lett. 32, 1611-1613 (1996).

42. S. LaRochelle, P.-Y. Cortes, H. Fathallah, L. A. Rusch, and H. B. Jaafar, "Writing and applications of fiber Bragg grating arrays," SPIE Proceedings 4087, 140-149 (2000).

43. P. Y. Cortes, F. Ouellette, and S. LaRochelle, "Intrinsic apodisation of Bragg gratings written using UVpulse interferometry," Electron. Lett. 34, 396-397 (1998).

44. H. G. Frohlich, and R. Kashyap, "Two methods of apodisation of fibre-Bragg-gratings," Opt. Commun. 157, 273-281 (1998) 
45. M. Shokooh-Saremi, V. G. Ta'eed, N. J. Baker, I. C. M. Littler, D. J. Moss, B. J. Eggleton, Y. L. Ruan, and B. Luther-Davies, "High-performance Bragg gratings in chalcogenide rib waveguides written with a modified Sagnac interferometer," J. Opt. Soc. Am. B 23, 1323-1331 (2006).

46. K. Finsterbusch, N. Baker, V. G. Ta'eed, B. J. Eggleton, D. Choi, S. Madden, and B. Luther-Davis, "Longperiod gratings in chalcogenide (As2S3) rib waveguides," Electronics Letters 42, 1094-1095 (2006).

47. D. Pudo, E. C. Magi, and B. J. Eggleton, "Long-period gratings in chalcogenide fibers," Opt. Express 14, 3763-3766 (2006).

48. I. C. M. Littler, L. B. Fu, E. C. Magi, D. Pudo, and B. J. Eggleton, "Widely tunable, acousto-optic resonances in Chalcogenide As2Se3 fiber," Opt. Express 14, 8088-8095 (2006).

49. B. J. Eggleton, P. A. Krug, L. Poladian, and F. Ouellette, "Long Periodic Superstructure Bragg Gratings in Optical Fibers," Electron. Lett. 30, 1620-1622 (1994).

50. N. J. Baker, H. W. Lee, I. C. Littler, C. M. d. Sterke, B. J. Eggleton, D.-Y. Choi, S. Madden, and B. Luther-Davies, "Sampled Bragg gratings in chalcogenide (As2S3) rib-waveguides," Opt. Express 14, 9451-9459 (2006).

51. J. Hubner, D. Zauner, and M. Kristensen, "Strong sampled Bragg gratings for WDM applications," IEEE Photon. Technol. Lett. 10, 552-554 (1998).

52. E. M. Vogel, M. J. Weber, and D. M. Krol, "Nonlinear Optical Phenomena in Glass," Physics and Chemistry of Glasses 32, 231-254 (1991).

53. M. E. Lines, "Oxide Glasses for Fast Photonic Switching - a Comparative-Study," J. Appl. Phys. 69, 68766884 (1991).

54. G. Lenz, J. Zimmermann, T. Katsufuji, M. E. Lines, H. Y. Hwang, S. Spalter, R. E. Slusher, S. W. Cheong, J. S. Sanghera, and I. D. Aggarwal, "Large Kerr effect in bulk Se-based chalcogenide glasses," Opt. Lett. 25, 254-256 (2000).

55. V. Mizrahi, K. W. Delong, G. I. Stegeman, M. A. Saifi, and M. J. Andrejco, "2-Photon Absorption as a Limitation to All-Optical Switching," Opt. Lett. 14, 1140-1142 (1989).

56. R. Jones, H. S. Rong, A. S. Liu, A. W. Fang, M. J. Paniccia, D. Hak, and O. Cohen, "Net continuous wave optical gain in a low loss silicon-on-insulator waveguide by stimulated Raman scattering," Opt. Express 13, 519-525 (2005).

57. T. K. Liang, and H. K. Tsang, "Nonlinear Absorption and Raman Scattering in Silicon-on-Insulator Optical Waveguides," IEEE J. Sel. Top. Quantum Electron. 10, 1149-1153 (2004).

58. M. Dinu, F. Quochi, and H. Garcia, "Third-order nonlinearities in silicon at telecom wavelengths," Appl. Phys. Lett. 82, 2954-2956 (2003).

59. H. K. Tsang, C. S. Wong, T. K. Liang, I. E. Day, S. W. Roberts, A. Harpin, J. Drake, and M. Asghari, "Optical dispersion, two-photon absorption and self-phase modulation in silicon waveguides at $1.5 \mathrm{mu}$ wavelength," Appl. Phys. Lett. 80, 416-418 (2002).

60. N. Sugimoto, H. Kanbara, S. Fujiwara, K. Tanaka, Y. Shimizugawa, and K. Hirao, "Third-order optical nonlinearities and their ultrafast response in Bi2O3-B2O3-SiO2 glasses," J. Opt. Soc. Am. B 16, 19041908 (1999).

61. H. C. Nguyen, K. Finsterbusch, D. J. Moss, and B. J. Eggleton, "Dispersion in nonlinear figure of merit of As2Se3 chalcogenide fibre," Electron. Lett. 42, 571-572 (2006).

62. M. N. Islam, C. E. Soccolich, R. E. Slusher, A. F. J. Levi, W. S. Hobson, and M. G. Young, "Nonlinear Spectroscopy near Half-Gap in Bulk and Quantum-Well Gaas/Algaas Wave-Guides," J. Appl. Phys. 71, 1927-1935 (1992).

63. M. Asobe, T. Ohara, I. Yokohama, and T. Kaino, "Low power all-optical switching in a nonlinear optical loop mirror using chalcogenide glass fibre," Electron. Lett. 32, 1396-1397 (1996).

64. O. P. Kulkarni, C. Xia, D. J. Lee, M. Kumar, A. Kuditcher, M. N. Islam, F. L. Terry, M. J. Freeman, B. G. Aitken, S. C. Currie, J. E. McCarthy, M. L. Powley, and D. A. Nolan, "Third order cascaded Raman wavelength shifting in chalcogenide fibers and determination of Raman gain coefficient," Opt. Express 14, 7924-7930 (2006).

65. K. S. Abedin, "Observation of strong stimulated Brillouin scattering in single-mode $\mathrm{As}_{2} \mathrm{Se}_{3}$ chalcogenide fiber," Opt. Express 13, 10266-10271 (2005).

66. K. Ogusu, H. P. Li, and M. Kitao, "Brillouin-gain coefficients of chalcogenide glasses," J. Opt. Soc. Am. B 21, 1302-1304 (2004).

67. D. P. Wei, T. V. Galstian, I. V. Smolnikov, V. G. Plotnichenko, and A. Zohrabyan, "Spectral broadening of femtosecond pulses in a single-mode As-S glass fiber," Opt. Express 13, 2439-2443 (2005).

68. P. V. Mamyshev, "All-optical data regeneration based on self-phase modulation effect," in Proceedings of European Conference on Optical Communication (ECOC)(Madrid, Spain, 1998), pp. 475-476.

69. M. Rochette, L. B. Fu, V. Ta'eed, D. J. Moss, and B. J. Eggleton, "2R optical regeneration: An all-optical solution for BER improvement," IEEE J. Sel. Top. Quantum Electron. 12, 736-744 (2006).

70. M. R. E. Lamont, L. B. Fu, M. Rochette, D. J. Moss, and B. J. Eggleton, "2R optical regenerator in $\mathrm{AS}(2) \mathrm{Se}(3)$ chalcogenide fiber characterized by a frequency-resolved optical gating analysis," Appl. Opt. 45, 7904-7907 (2006).

71. B. E. Olsson, P. Ohlen, L. Rau, and D. J. Blumenthal, "A simple and robust 40-Gb/s wavelength converter using fiber cross-phase modulation and optical filtering," IEEE Photon. Technol. Lett. 12, 846-848 (2000)

72. M. Asobe, "Nonlinear optical properties of chalcogenide glass fibers and their application to all-optical switching," Opt. Fiber Technol. 3, 142-148 (1997). 
73. M. R. E. Lamont, C. M. d. Sterke, and B. J. Eggleton, "Dispersion Engineering of Highly Nonlinear As2S3 Waveguides for Parametric Gain and Wavelength Conversion," in Australian Conference on Optical Fiber Technology (ACOFT)(Melbourne, Australia, 2007).

74. J. S. Sanghera, and I. D. Aggarwal, "Development of chalcogenide glass fiber optics at NRL," J. NonCryst Solids 213, 63-67 (1997).

75. R. G. DeCorby, N. Ponnampalam, M. M. Pai, H. T. Nguyen, P. K. Dwivedi, T. J. Clement, C. J. Haugen, J. N. McMullin, and S. O. Kasap, "High index contrast waveguides in chalcogenide glass and polymer," IEEE J. Sel. Top. Quantum Electron. 11, 539-546 (2005).

76. B. J. Eggleton, R. E. Slusher, C. M. deSterke, P. A. Krug, and J. E. Sipe, "Bragg grating solitons," Physical Review Letters 76, 1627-1630 (1996).

77. J. T. Mok, C. M. de Sterke, I. C. M. Littler, and B. J. Eggleton, "Dispersionless slow light using gap solitons," Nature Physics 2, 775-780 (2006)

78. C. Grillet, C. Smith, D. Freeman, S. Madden, B. Luther-Davis, E. C. Magi, D. J. Moss, and B. J. Eggleton, "Efficient coupling to chalcogenide glass photonic crystal waveguides via silica optical fiber nanowires," Opt. Express 14, 1070-1078 (2006).

\section{Introduction}

Chalcogenide glasses are truly remarkable materials and have been of enormous interest for infrared optics since the 1950's [1]. They are well known for their transparency far in to the infrared, and are widely used as infrared optical components. Apart from passive optical components, chalcogenide glass based optical devices have found a number of applications, particularly in fiber form [2,3] including: infrared optics and imaging; infrared laser power transmission; remote sensing and scanning near-field infrared microscopy.

However, for telecommunications based applications, these glasses stand out because they also exhibit third-order optical nonlinearities (Kerr, Raman and Brillouin) between two to three orders of magnitude greater than silica. This property has gained increasing recognition and has lead to a number of recent demonstrations of all-optical processes including switching [4], regeneration [5-7], wavelength conversion [8, 9], amplification [10], lasing [11], pulse compression [12], and slow light [13]. Photosensitivity to band-gap light is another important property that the chalcogenide glasses exhibit [14]. The photosensitivity of a chalcogenide glass is comparable only to the "hero" results observed in specially processed silica optical fiber [15] and is among one of its most striking properties. The nonlinear and photosensitive properties together offer numerous applications to a number of integrated alloptical functions.

Being a glass, the chalcogenides are a versatile platform. Apart from bulk optic components, chalcogenide glass fiber (both step index [16] as well as microstructured [17, 18]) and planar waveguide [19] devices have been developed. Importantly chalcogenide glasses can be formed with a variety of compositions and doped with additional elements to provide further functionality for example rare earth elements.

All of these features make chalcogenide glasses of significance in advancing the next generation photonic chip platform for ultrafast all-optical signal processing. The field however is still relatively new and so our purpose here is to provide an up-to-date review of the main features of chalcogenide glass photonics relevant to nonlinear waveguides aimed at researchers new to this material system. Thus this review paper covers some of the general properties of chalcogenide glasses as well as recent research highlights, including our more recent contributions to this emerging field.

The rest of the paper is structured as follows. First we present a general review of the main features and optical properties of the chalcogenide glasses. Topics covered include chemical structure, refractive index and dispersion, loss mechanisms, and waveguide fabrication. The following section reviews chalcogenide glass photosensitivity and applications for grating based waveguide function. The photosensitive characteristics are outlined and the main contributors to this area are reviewed. We focus on our recent results in demonstrating high quality, apodized Bragg gratings, long period gratings and sampled Bragg gratings. The next section examines the ultrafast nonlinear optical properties of the chalcogenide glasses, with an emphasis on the optical Kerr effect and the moderate effect of 
two-photon absorption. The impact of two-photon absorption in self-phase and cross-phase modulation (SPM and XPM) based devices is also numerically assessed. The following section reviews three chalcogenide glass device functions developed in our laboratory: signal regeneration, pulse compression and wavelength conversion. The final section briefly looks as how further device improvements can be achieved as well as other areas where chalcogenide glass based devices might have impact.

\section{Review of chalcogenide glasses}

\subsection{Chemical structure and properties}

Chalcogenide glasses are based on the chalcogen elements $\mathrm{S}$, Se and Te combined with network-forming elements, such as As, Ge, P, Sb, Si. The resulting glass exhibits a number of amorphous semiconductor like properties [20,21]. The glass structure consists of covalently bonded molecules, as opposed to the ionic bonding of other glasses, and these are weakly bound together via a combination of covalent and Van der Waals-like attraction [22, 23]. Chalcogenide glasses may form with a wide range of compositions as both hetropolar and homopolar bonds may form [21]. In the case of the As-S glass system, the atomic ratio of arsenic to sulfur can be varied between 1.5 and 9 [22]. As a result, the composition of the chalcogenide glasses can be adjusted to tune particular properties (e.g. refractive index, bandedge, nonlinearity). For a given chalcogenide glass system, increasing the relative atomic mass of the chalcogen or its proportion in the glass reduces the average bond strength. The weaker bonds of chalcogenide glasses contribute to them being generally less robust than oxide glasses. For example they have lower softening temperatures and hardness, and higher thermal expansion coefficients [1].

\subsection{Refractive index and dispersion}

Chalcogenide glasses possess relatively large refractive indices between 2 to greater than 3 [22]. Fig. 1 shows the refractive index and dispersion parameter for a common chalcogenide glass: $\mathrm{As}_{2} \mathrm{~S}_{3}$ [24]. The refractive index increases as sulfur is replaced by the more polarisable selenium and tellurium. The high refractive index is advantageous for strong optical field confinement which allows small waveguide bend radii (leading to compact circuit designs) and enhanced optical intensities (for efficient nonlinear interactions). In addition, the large index contrast relative to air can potentially provide a complete band-gap for photoniccrystals.

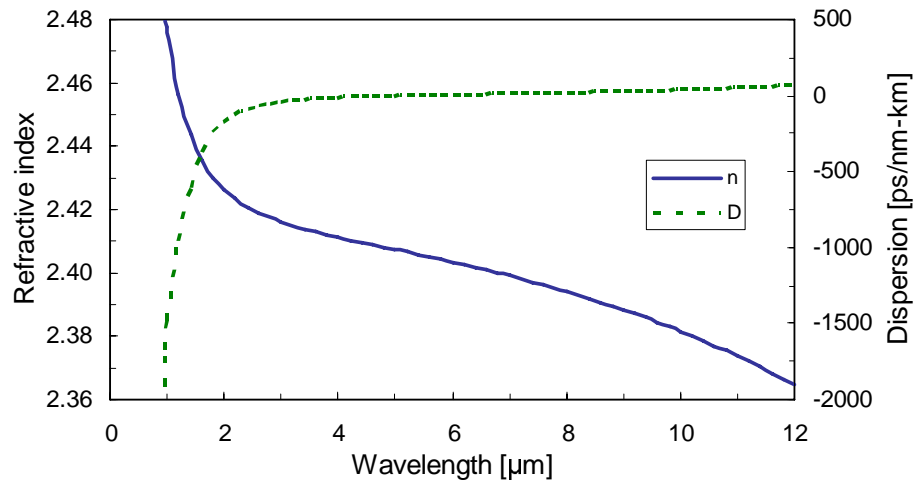

Fig. 1. Refractive index $(n)$ and dispersion parameter $(D)$ for arsenic tri-sulfide $\left(\mathrm{As}_{2} \mathrm{~S}_{3}\right)$ chalcogenide glass. [24]

The zero dispersion wavelength for chalcogenide glasses lies well in the mid IR (e.g. $5 \mu \mathrm{m}$ for $\mathrm{As}_{2} \mathrm{~S}_{3}$ ). At communication wavelengths around $1.55 \mu \mathrm{m}$ these materials exhibit strong normal dispersion (i.e. not anomalous). This is not necessarily detrimental to performance as typical device lengths are short, of the order of centimetres, and the dispersion sign ensures that 
modulation instability in nonlinear devices is eliminated [25]. Furthermore there is the possibility of using waveguide dispersion to engineer the chromatic dispersion, similar to what has been achieved in silicon [26].

\subsection{Linear loss mechanisms}

For chalcogenide glasses the long wavelength cut-off lies in the mid infrared due to large atomic masses and relatively weak bond strengths resulting in low phonon energies [20]. Fig. 2 compares the infrared transmission for several glasses, the last three being chalcogenide glasses [2]. As a rule of thumb, the transparency edge is $12 \mu \mathrm{m}$ for sulfide based glasses, $15 \mu \mathrm{m}$ for selenide glasses and $20 \mu \mathrm{m}$ for telluride glasses [21]. In the absence of extrinsic attenuation mechanisms, Rayleigh scattering defines the minimum attenuation of the glass within the electronic and multiphoton absorption window. Free carrier absorption is generally negligible in Chalcogenide glasses. While most chalcogenide glasses have small band gaps, they also exhibit low carrier mobility. As the magnitude of the free carriers in solids in the low mobility case is proportional to the carrier mobility, free carrier absorption is not important in chalcogenide glasses [1]. However chemical impurities, particularly oxygen can result in a drastic reduction in infrared transmission [1]. Typically ultra pure chemicals must be further purified, for example by hydrogen distillation, before being melted either under vacuum or within a nitrogen environment to create bulk glasses [21].

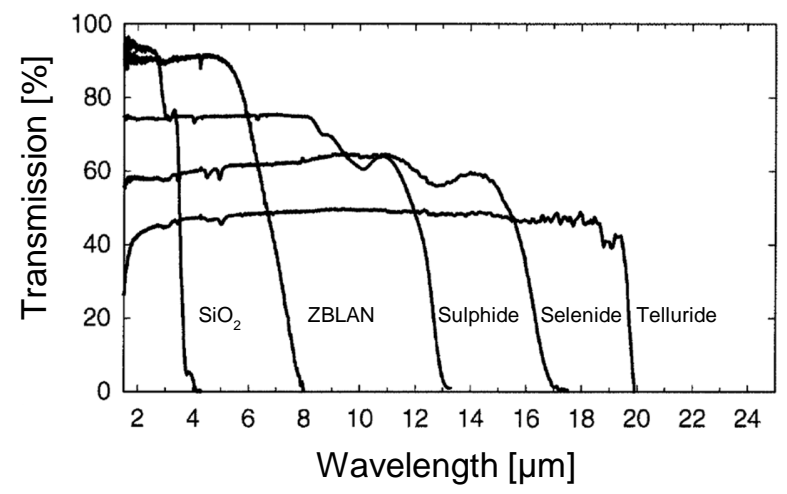

Fig. 2. Infrared transmission for several bulk glass samples (after reference [2]). Silicon, not shown in this figure, has a long wavelength cut-off at around $7 \mu \mathrm{m}[24]$.

\subsection{Waveguide fabrication}

Fiber fabrication has been achieved based on both the pre-form and double crucible techniques. In either case, low loss transmission is only achieved through careful management of impurities in the bulk. Multi-step purification processes have been developed at the Naval Research Laboratories [2]. More recent work has focused on microstructured chalcogenide glass fiber using the 'stack and draw' technique. [17, 18].

The simplest method for fabricating thin chalcogenide glass films is by thermal evaporation, however for multi-component systems, like chalcogenide glass, the differing evaporation rates of each element results in a change in the film stoichiometry poorly matching the bulk [21]. Sputter deposition tends to result in films with better stoichiometry though the deposition rate is relatively low [21]. Wet chemistry techniques have been used for precipitation of multi-component chalcogenide glasses compositions. Typically the glass is dissolved in an amine solvent (e.g. $n$-propylamine or diethylamine), spun onto a substrate and baked at low temperatures to remove solvent residue [27-31]. Recently thicker films have been achieved by spin coating of molten chalcogenide glass [32].

Chalcogenide glass films deposited by thermal evaporation, sputtering and wet chemical spin deposition all require a post-process anneal in order to bring about bulk glass like properties. Pulsed laser deposition of chalcogenide glass films has been proposed as a means 
of circumventing this step as the ablated materials has moderately high kinetic energy which can help densify the resulting films [33, 34]. More recently ultra-fast pulsed laser deposition has been developed [34] to overcome particulate contamination in the deposited films - a common problem with pulse laser deposition.

Significant progress has been made to applying the ultra-fast pulsed laser deposition technique to produce low loss chalcogenide glass waveguides [19]. Fig. 3 shows a scanning electron micrograph (SEM) of a typical rib waveguide cross section. A $2.5 \mu \mathrm{m}$ thick $\mathrm{As}_{2} \mathrm{~S}_{3}$ film was deposited via pulsed laser deposition [19] onto a silica-on-silicon substrate with a 2.5 $\mu \mathrm{m}$ thick thermal oxide layer acting as the bottom waveguide cladding. Rib waveguides were fabricated by photolithography and inductively coupled plasma reactive ion etching with $\mathrm{CF}_{4}$ $\mathrm{O}_{2}$ gas. Losses as low as $0.25 \mathrm{~dB} / \mathrm{cm}$ have been achieved using this technique [19].

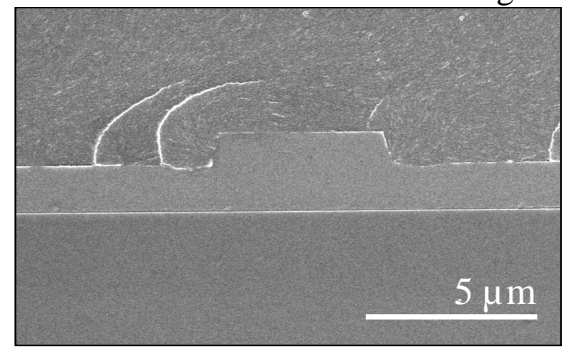

Fig. 3. Scanning electron micrograph cross-section of an $\mathrm{As}_{2} \mathrm{~S}_{3}$ rib waveguide.

\section{Photosensitivity}

\subsection{Photo-induced phenomena}

Chalcogenide glasses exhibit several types of photo-induced phenomena including: photocrystallisation, photo-polymerisation, photodecomposition, photo-contraction, photovaporisation, photo-dissolution of metals and light-induced changes in local atomic configuration. These changes are accompanied by changes in the optical band gap and thus optical constants (i.e. photo-darkening or photo-bleaching) [14,21]. Chalcogenide glasses exhibit strong photo-induced properties because of their inherent structural flexibility. The usually double covalent bonded chalcogen atom possesses a lone pair of non-binding electrons that under illumination can alter the bond number $[14,21]$. These photo induced effects have been used for the formation of various components including waveguides [35] and surface gratings [36].

\subsection{Bragg gratings and long period gratings}

The ability to write high quality gratings in chalcogenide glass waveguides via their photosensitivity [37] provides another advantage for these materials for photonic devices. The first demonstration was by Tanaka et al. using a Hill grating geometry [38]. Following this first milestone, Bragg gratings have been written using the side-writing technique into planar waveguides $[36,37,39,40]$, including studies of the growth dynamics. $\mathrm{As}_{2} \mathrm{~S}_{3}$ fiber gratings have also been achieved [41].

In terms of device quality gratings, the spectral response of chalcogenide gratings to date have largely been relatively basic, exhibiting no apodisation, poor uniformity, narrow rejection bandwidth, and weak transmission rejection. This may be in part due to the reliance on an uncompensated Mach-Zehnder interferometer which is particularly susceptible to environmental disturbances. This can affect the stability of the interference pattern and hence the grating quality. In contrast, a Sagnac interferometer provides excellent stability $[42,43]$ as both beams follow the same path in counter-propagating directions, ensuring that vibrations and air currents affect both beams equally, thereby minimizing phase distortion at the interference plane. By modifying the interferometer from a full Sagnac (i.e. shifting the writing plane away from the phase mask plane), sample placement is eased while still maintaining many of the desired stability features as each of the two interferometer beams 
travel a similar path. Using the setup shown in Fig. 4, we have achieved high quality $\mathrm{As}_{2} \mathrm{~S}_{3}$ waveguide Bragg gratings which exhibit sharp spectral features, wide rejection bands $(5 \mathrm{~nm}$ wide corresponding to an index change of 0.008) and deep transmission rejection $(33 \mathrm{~dB}$, limited by polarization mode leakage). Apodisation was accomplished through the short coherence length of the writing laser [44].

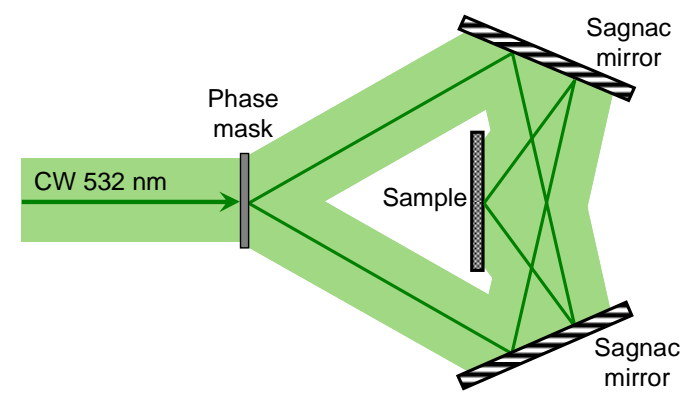

Fig. 4. Modified Sagnac grating writing interferometer. CW light from a frequency doubled $\mathrm{Nd}$ :YAG laser is split into two beams by a phase mask and reflected twice off two mirrors before interfering at the sample surface to create a photo-induced index change.

The Bragg gratings have also been used to determine the modal structure of the chalcogenide glass rib waveguides with significant success [45]. This in turn has allowed the design of long period gratings to couple the fundamental mode into a higher order mode at telecom wavelengths. Using a shadow mask technique we have achieved $17 \mathrm{~dB}, 15 \mathrm{~nm}(3 \mathrm{~dB})$ wide band rejection at a wavelength of $1525 \mathrm{~nm}$ [46]. We have also demonstrated nonphotosensitive long period gratings based on $\mathrm{As}_{2} \mathrm{Se}_{3}$ fiber using the micro-bend [47] and acousto-optic [48] techniques.

\subsection{Sampled gratings}

This success at long and short period Bragg gratings suggests that more complex gratings can be inscribed into the waveguides. One such grating that can benefit particularly well from the large refractive index change available in chalcogenides is a sampled Bragg grating (SBG) [49]. These provide a comb shaped transmission spectrum where the large index change available in chalcogenide glasses can extend the useable bandwidth and increase the strength beyond that possible in silica. SBGs open a new platform for WDM on-chip signal processing, providing a larger bandwidth response in a short, integrated device.

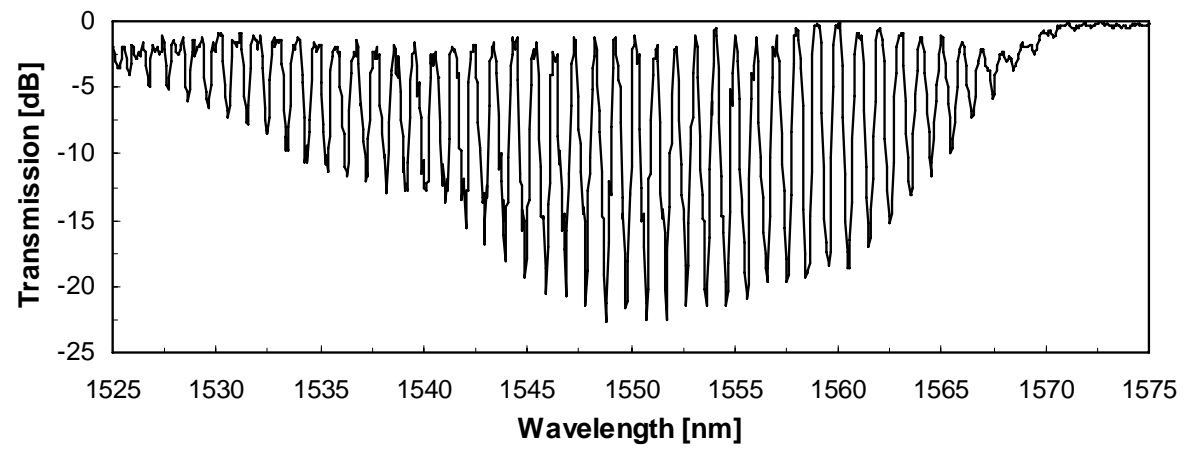

Fig. 5. Measured spectrum from an $\mathrm{As}_{2} \mathrm{~S}_{3}$ sampled Bragg grating [50].

A photo-lithographically etched chrome amplitude mask with periodic vertical slits was positioned directly in front of the waveguide to modulate the holographically written Bragg grating, resulting in a SBG [50]. The spectral response of a SBG written into an $\mathrm{As}_{2} \mathrm{~S}_{3}$ ribwaveguide is shown in Fig. 5. This spectrum has a wavelength separation of $0.966 \mathrm{~nm}$ and a 
3-dB bandwidth that spans almost $40 \mathrm{~nm}$, where the central 11 rejection peaks are each over $20 \mathrm{~dB}$ strong. To our knowledge the strength and bandwidth of this grating is comparable to the best results observed in optical fiber [49,51].

\section{Ultrafast optical properties of chalcogenide glasses}

\subsection{Nonlinear refraction and Raman processes}

Chalcogenide glasses have gained recent interest for their large third order nonlinear effects. Miller's rule [52], given by

$$
\chi^{(3)} \approx\left[\chi^{(1)}\right]^{4} 10^{-10}(e s u),
$$

is a particularly simple method for estimating the third order nonlinearity. From the large refractive indices for the chalcogenide glasses ( 2 to $>3$ ) it is apparent that Miller's empirical rule predicts nonlinear coefficients approximately two orders of magnitude larger than for silica. Other semi-empirical models [22, 52] also show a similar correlation between refractive index and nonlinearity, including a semi-quantitative model based on bond-orbital theory [53]. The results of this work have been found to be in good agreement with known data for a range of glasses, and indicate that strong nonlinearity can be expected from chalcogenide glasses [10, 54]. In fact, depending on composition and measurement wavelength, the tabulated nonlinear refractive index of chalcogenide glasses [22, 52] is indeed between two to three orders of magnitude greater than of fused silica. The nonlinear refractive indices for $\mathrm{As}_{2} \mathrm{~S}_{3}$ and $\mathrm{As}_{2} \mathrm{Se}_{3}$ are $2.92 \times 10^{-18}$ and $1.2 \times 10^{-17} \mathrm{~m}^{2} / \mathrm{W}$, which normalized to the silica value of $2.2 \times 10^{-20} \mathrm{~m}^{2} / \mathrm{W}$ is approximately 130 and 540 respectively $[19,54]$.

Most of the Kerr nonlinear effect is associated with the ultra-fast electronic processes with time scales on the order of $50 \mathrm{fs}$ [10]. Measurements have shown that the nuclear contribution to the nonlinear effect for sulfide based glasses is between $12 \sim 13 \%$ [22]. The measured nuclear time scale, for selenide glasses, is on the order of $500 \mathrm{fs}$ [10]. As the nuclear contribution ratio is similar to that of silica, the Raman gain coefficient is also several orders of magnitude larger than silica $[10,25]$. It is also important to note that the ultrafast response time of these nonlinearities allows high bit rate signal processing applications without resorting to differential interferometer operation as typically used in semiconductor optical amplifier based devices.

\subsection{Two-photon absorption and the nonlinear figure of merit}

Multi-photon absorption is a nonlinear process that occurs at harmonics of the electronic band absorption frequency causing two or more photons to be simultaneously absorbed. The lowest order effect, two-photon absorption (TPA), is described by the imaginary part of the third order susceptibility resulting in an intensity dependent loss:

$$
\frac{d I}{d z}=-\alpha I-\alpha_{2} I^{2}
$$

Here $I$ is the optical intensity as a function of propagation distance $z, \alpha$ is the linear absorption coefficient and $\alpha_{2}$ is the TPA coefficient. In the context of all-optical nonlinear devices based on the Kerr coefficient, TPA is generally observed to be a nuisance, leading not only to a decrease in transmission, but also an increase in the switching threshold [55]. Furthermore, in some materials the free carriers generated by TPA can slow the response of nonlinear interactions. For example TPA induced free carriers in silicon typically have 10-20 nanosecond life times and require the introduction of $\mathrm{p}$-i-n diode structures in order to reduce this to the 1 nanosecond range [56]. While free carriers are not important in chalcogenide glasses, the direct effect of TPA must also be examined.

The nonlinear figure of merit (FOM), a geometry-independent criteria [55] used to classify different nonlinear materials and widely adopted in the literature, is defined as:

$$
\mathrm{FOM}=n_{2} / \alpha_{2} \lambda>1
$$

where $n_{2}$ is the Kerr coefficient and $\lambda$ is the optical wavelength. Fig. 6 (a) shows the transmission as a function of normalized power for varying nonlinear FOM. For low values 
of the nonlinear FOM this results in saturation type behaviour. Fig. 6(b) shows the FOM as a function of nonlinear transmission for a device requiring a total nonlinear phase shift of $\pi \mathrm{rad}$ [54]. From this example, it is apparent that a nonlinear FOM of $>2$ is required to ensure less than 20\% transmission loss. Assuming that linear propagation losses are low, the goal for third-order nonlinear materials is then to maximize the Kerr coefficient while minimizing the effect of TPA [54].
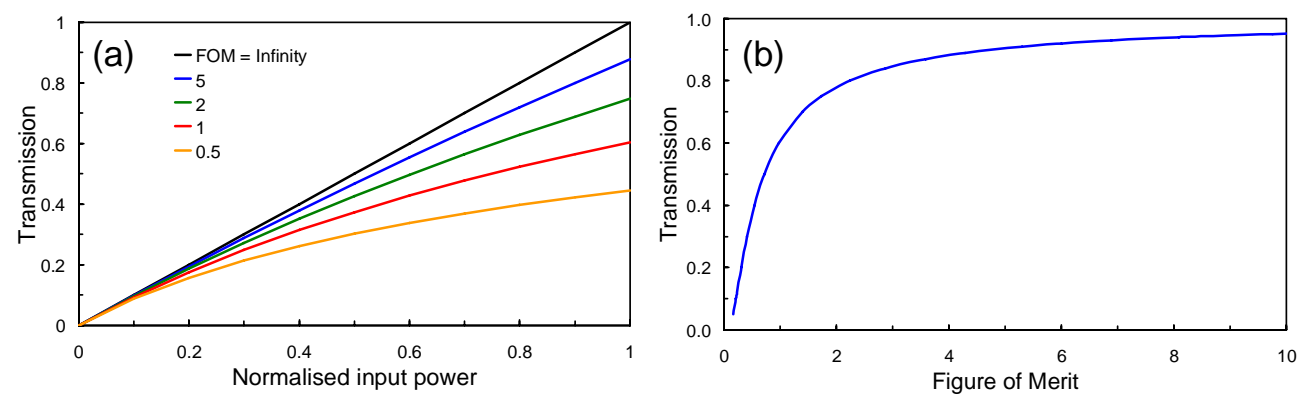

Fig. 6. (a) Numerical simulation of TPA induced transmission loss for varying nonlinear FOM. For no TPA $(\mathrm{FOM}=\infty)$, the total nonlinear phase shift is $2 \pi$. (b) Nonlinear transmission versus FOM for $\pi$ phase shift. [54]

Apart from modifying the device transmission behaviour, TPA also reduces the spectral broadening that occurs through SPM and XPM. Fig. 7 shows the typically SPM spectral response of an intense pulse propagating through a medium (a) without TPA and (b) with TPA corresponding to FOM $=0.5$. Essentially the additional loss reduces the effective interaction length, thus reducing the total nonlinear phase shift.
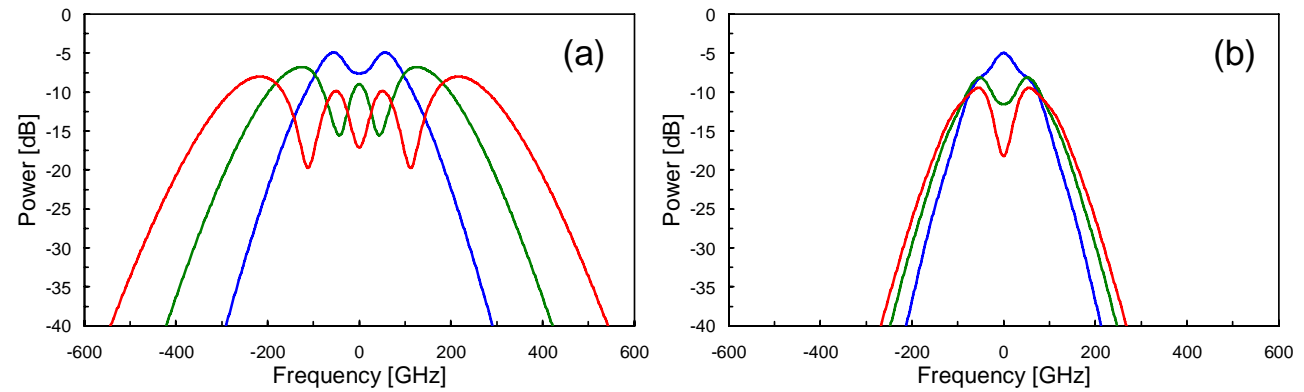

Fig. 7. Numerical simulation of self phase modulation spectral broadening of pulses. (a) Without two-photon absorption $(\mathrm{FOM}=\infty)$, and $(\mathrm{b})$ with two-photon absorption $(\mathrm{FOM}=0.5)$.

As amorphous materials (e.g. glasses) do not exhibit a clearly defined band-edge, finite twophoton absorption is expected even at wavelengths longer than the half band-gap energy [54]. The purity of the glass largely determines the tail of the absorption edge, and thereby also strongly affects the level of two-photon absorption [10]. As chalcogenide glasses generally have a band gap that falls in the visible region of the electromagnetic spectrum, they tend to exhibit low to occasionally moderate levels of TPA at C-band communication wavelengths. The FOM of $\mathrm{As}_{2} \mathrm{~S}_{3}$ and $\mathrm{As}_{2} \mathrm{Se}_{3}$ is $>10$ and $\sim 2$ in the near infrared [19, 54]. For comparison, silica having a band-edge in the UV, does not exhibit two-photon absorption near infrared wavelengths, while the FOM of silicon is $\sim 0.4$ [57-59]. Table 1 shows a comparison of the nonlinear index, two photon absorption coefficient and nonlinear FOM for several third-order nonlinear materials. 
Table 1. Comparison of nonlinear optical properties of several third-order nonlinear materials at $\lambda=1.5 \mu \mathrm{m}$. $[25,58,60]$

\begin{tabular}{lccc}
\multicolumn{1}{c}{ Material } & $n_{2}\left[\times 10^{-20} \mathrm{~m}^{2} / \mathrm{W}\right]$ & $\alpha_{2}\left[10^{-12} \mathrm{~m} / \mathrm{W}\right]$ & FOM \\
\hline Chalcogenide glass $\left(\mathrm{As}_{2} \mathrm{~S}_{3}\right)$ & 290 & $<0.01$ & $>10$ \\
Chalcogenide glass $\left(\mathrm{As}_{2} \mathrm{Se}_{3}\right)$ & 1200 & 1 & 2 \\
Bismuth Oxide $\left(\mathrm{Bi}_{2} \mathrm{O}_{3}\right)^{*}$ & 110 & - & - \\
Silicon $(\mathrm{Si})$ & 440 & 8.4 & 0.4 \\
Silica $\left(\mathrm{SiO}_{2}\right)^{*}$ & 2.2 & - & - \\
\hline
\end{tabular}

* The large optical band-gap of bismuth oxide and silica results in negligible two photon absorption at these wavelengths.

Nonlinear transmission and SPM spectral broadening can be used to infer the nonlinear index and two-photon absorption coefficient through comparison with simulations numerically solving the nonlinear Schrödinger equation. We have used this technique with an optical parametric oscillator (OPO) to characterize the wavelength dependence of the nonlinear FOM transmission for $\mathrm{As}_{2} \mathrm{Se}_{3}$ fiber [61]. Fig. 8 shows the characteristic increase in the nonlinear coefficients as the photon energy of the operating wavelength approaches the first sub-harmonic of the electronic band-gap, with the optimal photon energy being just under the half band-gap in order to optimize the nonlinear figure of merit [10]. By adjusting the composition of the chalcogenide glass and thus shifting the band-edge it is possible to engineer the nonlinear absorption - similar to what has been achieved in semiconductors (e.g. Al-Ga-As) [62].
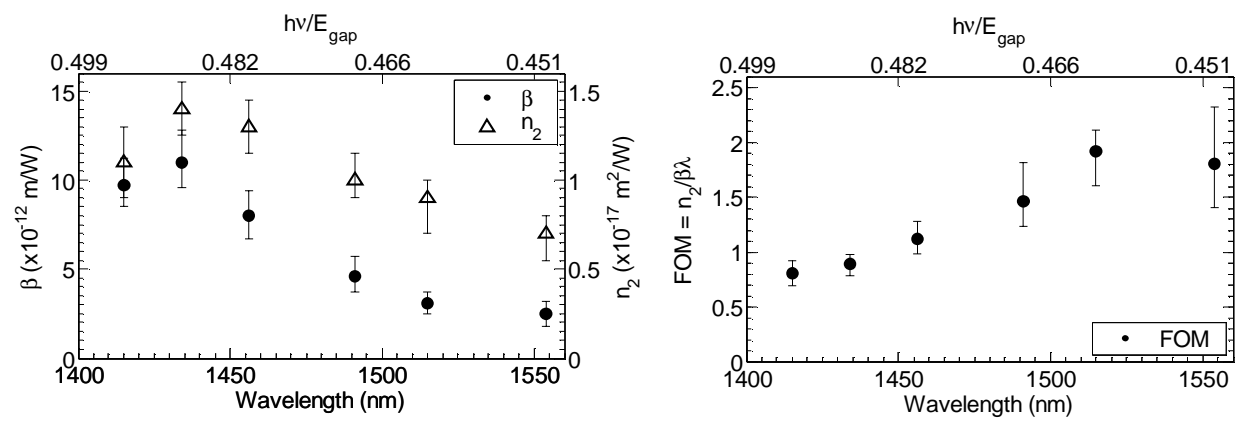

Fig. 8. Dispersion of (a) $n_{2}$ and $\beta$, (b) the nonlinear figure of merit for As2Se3.

\section{All-optical signal processing}

Large refractive indices, a high nonlinearity and moderate to low two-photon absorption make chalcogenide glasses ideal for demonstrating integrated devices for all-optical signal processing. Initial pioneering work by Asobe et al [63], demonstrating a switch based on a nonlinear optical loop mirror, has been followed in recent years by a significant increase in the number of papers in this field, ranging from examining Raman $[10,64]$ and Brillouin $[65$, 66] processes to actual signal processing functions and device demonstrations [5-7, 9, 12, 13, 67]. In line with the previous sections, here we focus on the Kerr nonlinearity as well as the role of two-photon absorption by examining chalcogenide glass 2R SPM based optical regeneration, SPM based pulse compression and XPM based wavelength conversion. 


\section{$5.12 R$ regeneration (fiber \& waveguide)}

A particularly interesting application of chalcogenide glass in all-optical signal processing is signal regeneration. We have investigated the feasibility of using $\mathrm{As}_{2} \mathrm{Se}_{3}$ chalcogenide glass fiber for all-optical signal regeneration based on SPM followed by spectral filtering [68]. In this scheme a noisy return-to-zero input signal is passed through a nonlinear, dispersive medium producing SPM induced spectral broadening. Low power noise experiences only little SPM spectral broadening, and so is filtered out by the band-pass filter, which is offset from the input centre wavelength, whereas pulses experience sufficient spectral broadening to be partially transmitted through the band pass filter. This results in a step-like power transfer function, which has been shown to improve the Q-factor and bit error rate (BER) for modulated signals at $40 \mathrm{~Gb} / \mathrm{s}$ [69]. Dispersion is critical in determining the proper shape the nonlinear transfer function. Without dispersion, large oscillations would appear on the broadened spectrum which, after filtering, manifest as oscillations in the transfer function. In contrast, a small but significant amount of normal dispersion reduces the spectral oscillations, resulting in a step-like transfer function [68].

In our investigations we have utilized single mode $\mathrm{As}_{2} \mathrm{Se}_{3}$ chalcogenide glass fiber as the nonlinear medium for pulse regeneration. The fiber has an effective area of $A_{\text {eff }}=37 \mu \mathrm{m}^{2}$, yielding a nonlinearity coefficient of $\lambda \sim 1200 \mathrm{~W}^{-1} \mathrm{~km}^{-1}$, where $\gamma=2 \pi n_{2} / \lambda A_{\text {eff. }}$. We measured the group delay of a $2.8 \mathrm{~m}$ length of $\mathrm{As}_{2} \mathrm{Se}_{3}$ fiber using the differential phase shift method, obtaining a dispersion of $D \sim-500 \mathrm{ps} / \mathrm{nm} / \mathrm{km}$ at $1550 \mathrm{~nm}$. As outlined earlier, the dispersion at telecommunication wavelengths is not only much larger than standard single mode fiber $(+17 \mathrm{ps} / \mathrm{nm} / \mathrm{km})$ but the negative sign of the chalcogenide dispersion is appropriate for SPMbased regeneration.

The data points in Fig. 9(a) correspond to the measured nonlinear power transfer function. Output power limiting function at $\sim 8 \mathrm{~W}$ peak input power, as well as threshold behaviour is clearly evident. The former is effective in suppressing the noise in the logical ones whilst the latter contributes to suppressing noise in the zeros. Residual oscillations remain at high peak power levels but this is smaller than what would result without the presence of the large normal dispersion. Fig. 9(a) also shows the resulting theoretical power transfer curves for FOM ranging from infinite to 0.5. Materials with a FOM in the range of 15 produce a flatter power transfer function than those with no TPA (infinite FOM). For FOM $<1$, the transfer curves lose their sharp edge characteristics and round off. The experimental data agrees well with the theoretical curve that has FOM $\sim 2.5$. This value is close to the experimental value of $\mathrm{FOM} \sim 2.0$ for $\mathrm{As}_{2} \mathrm{Se}_{3}$ as measured by the nonlinear transmission experiment [61].
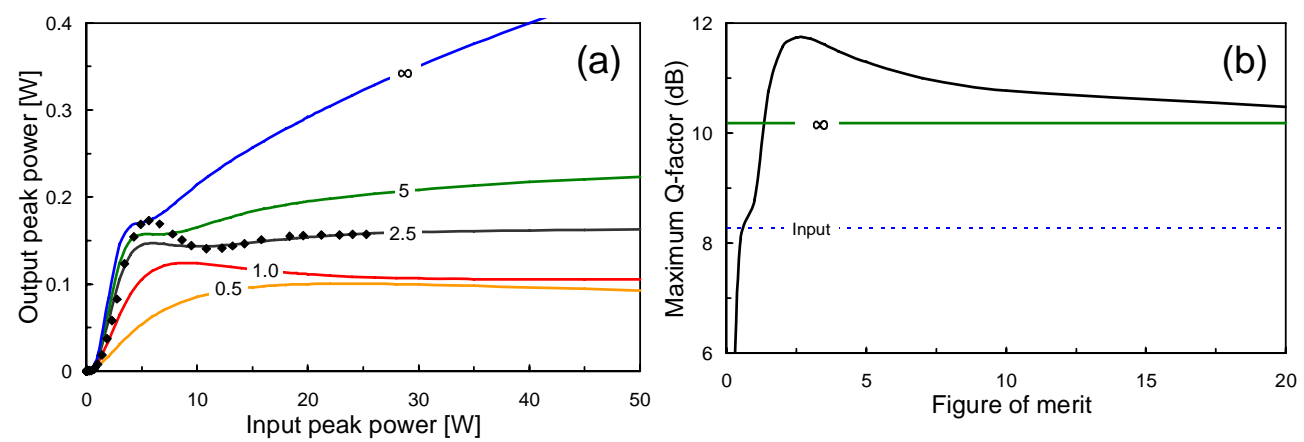

Fig. 9. (a) Calculated power transfer curves for $2 \mathrm{R}$ regenerator for different FOM with fixed $n_{2}$, including experimental data for a device with an FOM of $\sim 2.0$ (black diamonds). (b) Q-factor at the output of the $2 \mathrm{R}$ optical regenerator as a function of FOM (optimal input power use at each FOM). The dashed horizontal solid line is the input Q-factor while the solid horizontal line represents the output Q-factor for a device with no TPA (infinite FOM). 
To better understand the performance of the $2 \mathrm{R}$ regenerator in a system, we simulated a pseudorandom bit sequence at $40 \mathrm{~Gb} / \mathrm{s}$ with an return-to-zero signal including ASE noise (Optical signal to noise ratio $=11 \mathrm{~dB})$ [70]. Fig. 9(b) summarizes these results by plotting the maximum optimized (with respect to input peak power) output Q-factor as a function of FOM. It shows that even a small amount of TPA improves device performance, although for very small FOM the output Q-factor is actually less than the input Q-factor since devices with very large TPA will degrade signal quality. There is clearly an optimum range of FOM, from 2 to 4 , where the improvement in Q-factor is maximized. These results are in contrast with nonlinear switching where TPA cannot improve device performance. It is interesting to note that $\mathrm{As}_{2} \mathrm{Se}_{3}$ chalcogenide glass has a nearly optimum nonlinear $\mathrm{FOM}$ for $2 \mathrm{R}$ regeneration.

In order to further reduce the device size we have examined a photonic integrated chip based regenerator design. Here we have used a low-loss, $5 \mathrm{~cm}$ long, $\mathrm{As}_{2} \mathrm{~S}_{3}$ rib waveguide similar to Fig. 3. Two photosensitive Bragg gratings were inscribed successively at the end of the waveguide using the techniques outlined earlier. This formed the band-pass filter for the SPM regeneration scheme (Fig. 10(b)). $\mathrm{As}_{2} \mathrm{~S}_{3}$ has a lower nonlinearity than $\mathrm{As}_{2} \mathrm{Se}_{3}$ and also exhibits a FOM > 10, consequently this device does not utilize any nonlinear absorption to saturate the nonlinear transfer function. Rather it depends on the conventional limiting effects of a SPM regenerator (similar to a silica fiber embodiment) [68]. Fig. 10 shows the experimental spectral results for the propagation of 1.4 ps pulses. Fig. 10(a) shows the pulse spectra versus power for though a bare waveguide with $1.5 \pi$ radians of nonlinear phase shift produced using $\sim 50 \mathrm{~W}$ peak power. Fig. 10(c) shows the pulse spectra at the output of the photonic chip based regenerator while part (d) shows the resulting power transfer function.

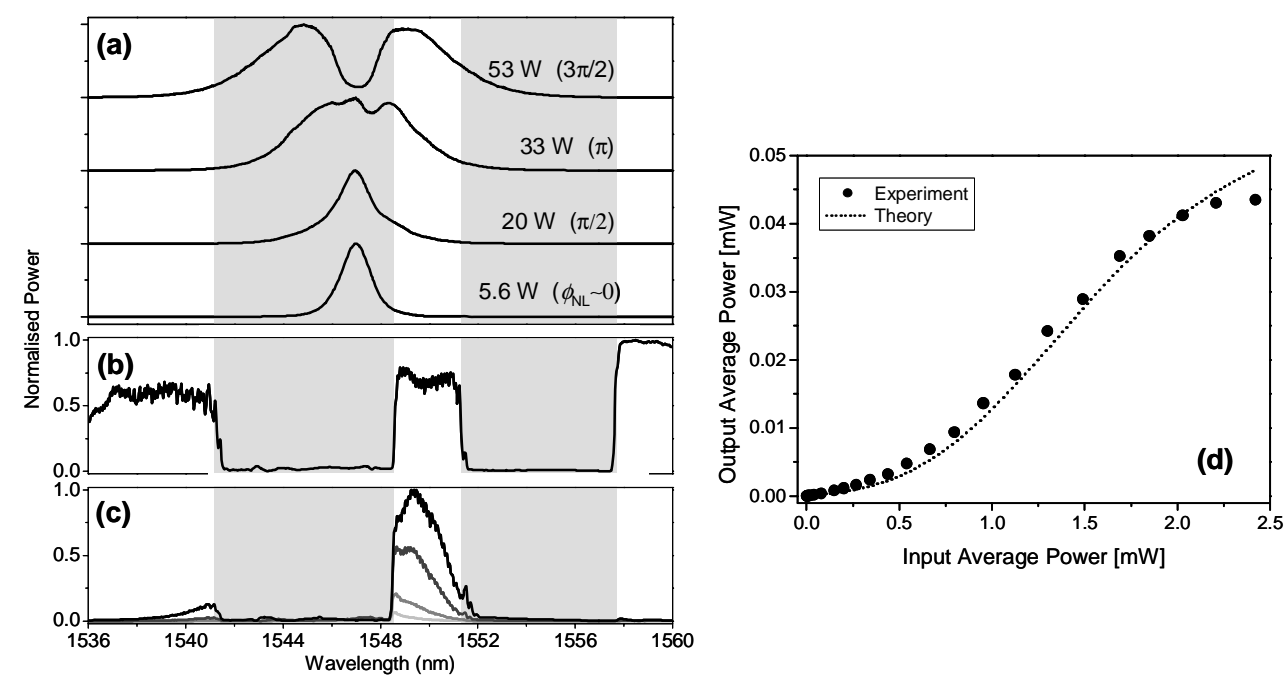

Fig. 10. (a) Evolution of pulse spectra versus power through a bare waveguide with no grating filter, showing spectral broadening due to SPM. (b) Transmission spectrum of the band-pass filter (formed by two sequential offset gratings) for TE polarized light, showing a pass band of $2.8 \mathrm{~nm}$ near $1555.0 \mathrm{~nm}$, offset by $3 \mathrm{~nm}$ from the carrier wavelength. (c) Sliced SPM broadened output spectra after the filter. (d) Resulting nonlinear power transfer curve for the integrated regenerator.

\subsection{Pulse compression (fiber)}

Apart from optical regeneration, the strong normal dispersion of chalcogenide glass combined with the high optical nonlinearity can also be exploited in compact, low-power, high quality optical pulse compression schemes. First the high optical nonlinearity and dispersive properties are used to generate a SPM broadened pulse with a linear frequency chirp at low peak powers. The chirped pulses can then be efficiently compressed using a precisely tailored, chirped fiber Bragg grating. 
In our experiments, we used a figure-eight fiber laser as a pump source, producing $6 \mathrm{ps}$ pulses at $9 \mathrm{MHz}$ repetition rate and $1550 \mathrm{~nm}$ centre wavelength. The laser was butt-coupled to a $4.1 \mathrm{~m}$ long $\mathrm{As}_{2} \mathrm{Se}_{3}$ chalcogenide fiber. The peak input power launched into the fiber was estimated to be $P=35 \mathrm{~W}$. Fig. 11 shows the broadened spectrum following the nonlinear propagation, compared to the original laser pulse. The dashed line is the result of the numerical simulations whereas the solid line represents the retrieved frequency resolved optical gating (FROG) data. Note the spectrum has been broadened substantially. In contrast to spectral broadening by SPM in the absence of dispersion where the spectrum is highly modulated with spectral nulls the spectral intensity in the case of this high dispersion is smooth across the whole bandwidth. Moreover, the interplay between SPM and a highly positive dispersion generates an almost perfectly quadratic phase, equivalent to a linear frequency chirp.

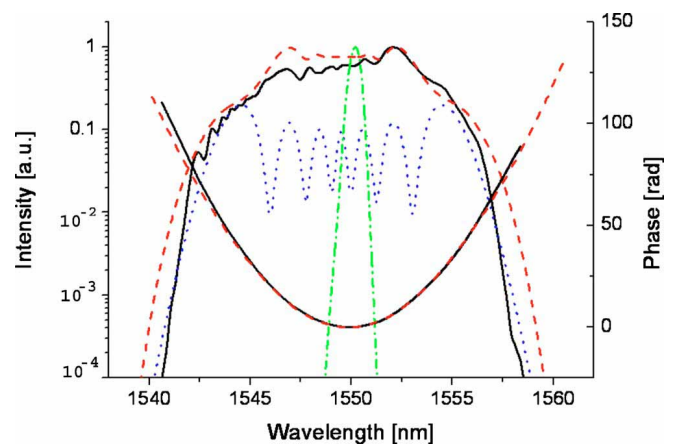

Fig. 11. Spectral intensity and phase of the laser pulses after the chalcogenide fiber. Solid line: retrieved from the FROG measurements. Dashed line: Numerical simulations. Dotted: Simulations excluding GVD showing high spectral modulation. Dash-dot: input spectrum.

Based on the FROG measurements, we designed a suitable fiber Bragg grating with a chirp matched to (but with opposite sign) the chirp on the laser pulses. The grating was written with a phase mask into a silica cladding mode suppressed fiber with $\Delta n=10^{-3}$ and a length of about $4 \mathrm{~mm}$. Fig. 12 shows the temporal intensity and phase of the pulses after the grating. We achieve a compressed pulse duration of as short as $420 \mathrm{fs}$ with an almost constant phase across the main part of the pulse, i.e., the pulse is almost transform limited.

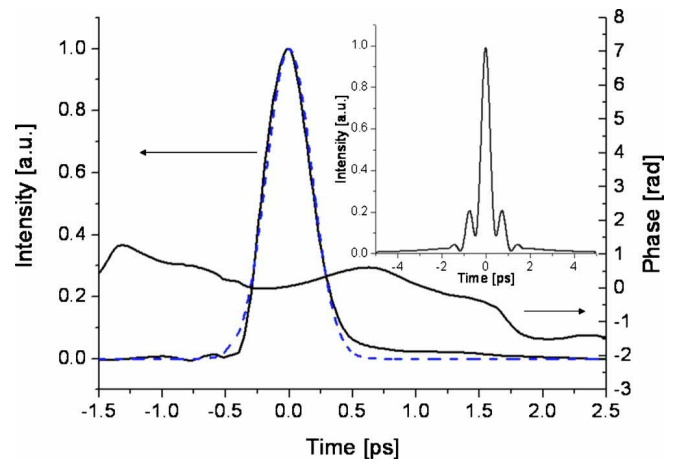

Fig. 12. Experimentally generated temporal intensity and phase of the compressed pulses, together with a Gaussian fit to the intensity (dotted line). The inset shows the temporal intensity of the compressed pulse without high GVD in the fiber leading to a considerable pulse pedestal and high temporal sidelobes.

\subsection{Wavelength conversion}

The SPM regenerator concept can easily be extended to other important all-optical functions such as wavelength conversion. We have investigated the feasibility of wavelength conversion in chalcogenide glass waveguides via cross phase modulation (XPM) with side band filtering 
$[8,9]$. Fig. 13 shows the principle of operation: a continuous wave $(\mathrm{CW})$ probe experiences XPM from co-propagating signal pump pulses, which generates optical sidebands. This is converted to amplitude modulation by using a band pass filter to select a single sideband [71].
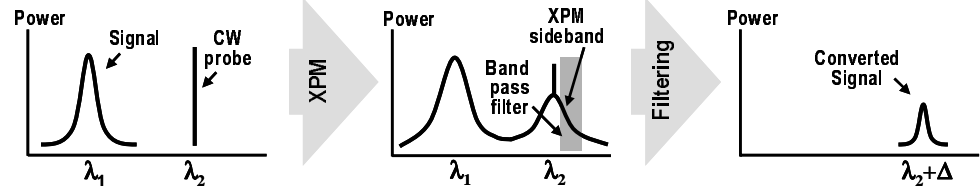

Fig. 13. Principle of XPM wavelength conversion. Amplified pulsed pump signal (at $\lambda_{1}$ ) imposes a nonlinear frequency chirp onto a co-propagating wavelength tunable $\mathrm{CW}$ probe (at $\lambda_{2}$ ) through the nonlinear refractive index. Filtering one of the XPM generated sidebands results in wavelength conversion (to $\lambda_{2}+\Delta$ ).

Fig. 14 shows schematically the experimental system used to demonstrate wavelength conversion. The $1 \mathrm{~m}$ length of single mode $\mathrm{As}_{2} \mathrm{Se}_{3}$ fiber had similar specifications to the fiber used in the SPM regenerator experiment. The signal data consisted of $7 \mathrm{ps}$ pulses from a $10 \mathrm{GHz}$ laser modulated with a pseudo-random bit-stream data from the bit error rate (BER) test bed system. The amplified optical signal was combined with a $\mathrm{CW}$ probe from a wavelength tunable amplified laser diode and coupled into the $\mathrm{As}_{2} \mathrm{Se}_{3}$ fiber. The output of the $\mathrm{As}_{2} \mathrm{Se}_{3}$ fiber was then sent through a $0.56 \mathrm{~nm}$ tunable grating filter offset to longer wavelengths to remove the pump and select a single XPM sideband. An in-line, $200 \mathrm{pm}$ wide, fiber Bragg grating (FBG) notch filter was used to further suppress the residual $\mathrm{CW}$ carrier.

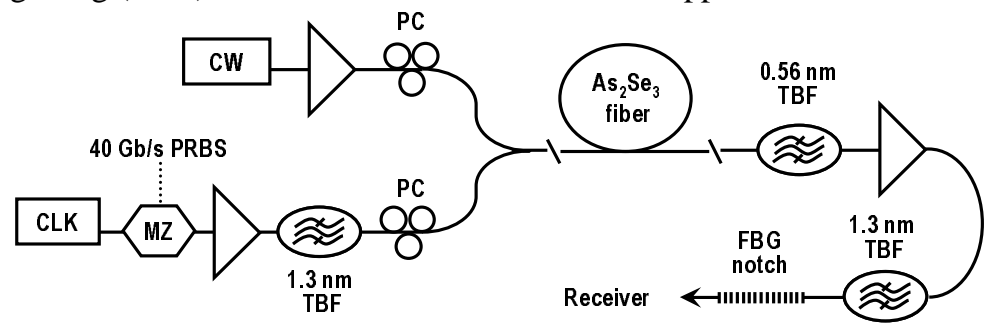

Fig. 14. System setup for demonstrating wavelength conversion. CLK: $10 \mathrm{GHz}$ actively mode locked, fiber laser, FBG notch: fiber Bragg grating notch filter, MZ: Mach-Zehnder modulator, PC: polarization controlled, PRBS: pseudo-random bit sequence, TBF: tunable band pass filter.

We demonstrated wavelength conversion over a $10 \mathrm{~nm}$ range using $2.1 \mathrm{~W}$ of peak power [9]. System measurements showed a penalty of $\sim 1.4 \mathrm{~dB}$ at a BER $=10^{-9}$ for conversion to three different wavelengths. The relative group velocity difference between the pump and probe (i.e. walk-off) reduced the amount of bandwidth generated through XPM as a function of wavelength offset, limiting the range of wavelength conversion.

In order to further reduce device size and extend the wavelength range we also investigated a planar waveguide based wavelength converter with a smaller effective core area. Pulses from a passively mode-locked figure-eight laser (pulse width $5.4 \mathrm{ps}$, repetition rate $9 \mathrm{MHz}$ ) were amplified and combined with a $\mathrm{CW}$ probe from a wavelength tunable amplified laser diode. The combined pump and probe were coupled into the rib waveguide. The output of the $\mathrm{As}_{2} \mathrm{~S}_{3}$ waveguide was then sent through a $1.3 \mathrm{~nm}(3-\mathrm{dB})$ tunable band-pass filter offset from the $\mathrm{CW}$ probe wavelength and the residual $\mathrm{CW}$ carrier was blocked using a $200 \mathrm{pm}$ wide fiber Bragg grating (FBG) notch filter.

Fig. 15 (a) shows the spectra directly after the waveguide of the unfiltered output signal containing both the pump ( $2 \mathrm{~nm}$ wide signal at $1549.7 \mathrm{~nm})$ and CW probes set to three different wavelengths. The sidebands on each probe are clearly visible. Note that the strength of the sidebands are proportionately reduced because of the extremely low duty cycle (5.4 ps pulses at $9 \mathrm{MHz}$ ) used in these experiments. Also shown in Fig. 15 (b) are the converted pulse spectra. Unlike the fiber based experiment, the XPM spectral broadening generated within the waveguide for a $\mathrm{CW}$ probe set to $1563.7 \mathrm{~nm}$ did not vary significantly from when the probe was set to $1555.5 \mathrm{~nm}$. While material dispersion parameter of $\mathrm{As}_{2} \mathrm{~S}_{3}$ is still significant 
$(-400 \mathrm{ps} / \mathrm{km} \mathrm{nm}$ at $1550 \mathrm{~nm}$ [72]) the $5 \mathrm{~cm}$ device length ensured that walk-off was minor, even for large pump-probe offsets. The functional conversion bandwidth of this device was calculated to be $100 \mathrm{~nm}$.

Frequency resolved optical gating (FROG) measurements determined that the output pulse experiences very little temporal amplitude distortion over the input while the temporal phase experiences a small degree of quadratic distortion. The effect of this on the output spectra, however, is modest; the induced chirp results in spectral broadening on the order of $24 \%$ - still close to transform limited.
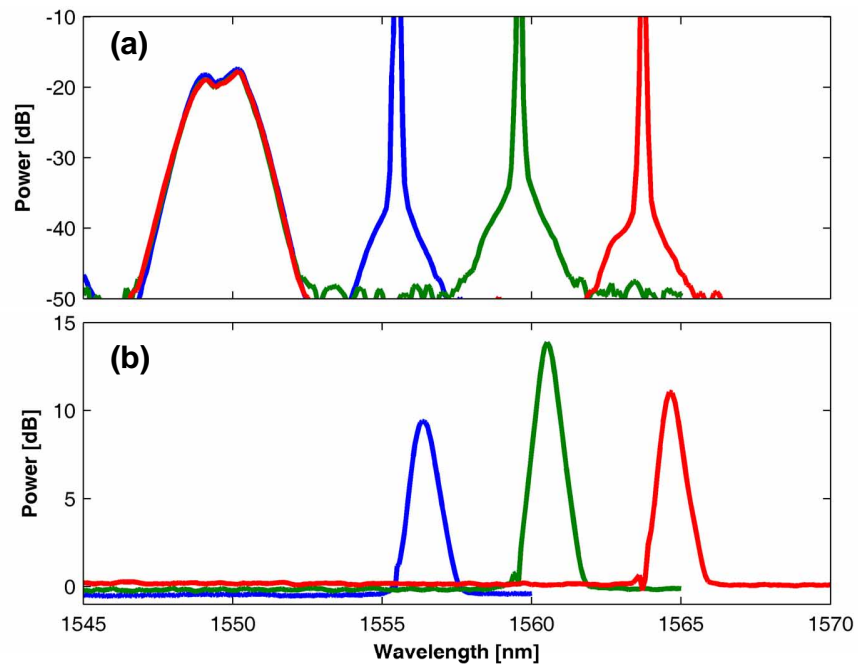

Fig. 15. (a) Unfiltered output spectra of signal from waveguide showing both pulsed pump and three different CW probes with XPM sidebands imprinted on them. (b) Experimental filtered output spectra of device leaving behind only a single sideband.

\section{Summary and future prospects}

In this article we have reviewed the major features of chalcogenide glasses and focused on exploring the Kerr nonlinearity including the impact of two photon absorption. We show that chalcogenide glasses offer several important properties for ultrafast all-optical signal processing: large refractive index, photosensitivity, high nonlinearity, low two photon absorption, fast response time and the flexibility of glass composition. These properties have so far allowed us to demonstrate three device functionalities: $2 \mathrm{R}$ signal regeneration, pulse compression and wavelength conversion. By reducing waveguide cross sectional areas, increasing interaction lengths, optimizing glass chemistry and reducing overall loss, further improvements in device performance is expected.

Preliminary work indicates that waveguide dispersion can be readily engineered, as has been recently achieved in silicon nanowires $[26,73]$. While the regenerator performance improves for large values of normal dispersion, this is not so for other processes. Examples include devices based on cross phase modulation (particularly for short pulses in long waveguides) and four wave mixing based devices.

Chalcogenide glasses do suffer from some potential issues related to stability and aging. While mechanically weaker than silica based glasses, the chalcogenide glass compositions are themselves stable. Note that the US Naval Research Laboratories in Washington DC has been working with these glasses for many years [74]. The optical damage mechanisms are not well understood, though certain compositions do offer greater resistance than others. We have, however, operated $\mathrm{As}_{2} \mathrm{~S}_{3}$ chalcogenide glass planar waveguides stably at continuous average intensities at $1550 \mathrm{~nm}$ of $>6 \mathrm{MW} / \mathrm{cm}^{2}$, as well as peak pulse intensities $\approx 1 \mathrm{GW} / \mathrm{cm}^{2}$, which is more than sufficient for most applications. 
While current research is focused on the chalcogenide and silicon platforms, the potential also lies in devices integrating both materials in a photonic chip hetrostructure [75]. Such a device could utilize the advantages of both media - for instance electrical-optical interactions mediated by free carrier effects in silicon waveguides, while nonlinear-optical processes would be based on chalcogenide glass waveguides. It remains be seen how viable such a prospect is. Other areas that chalcogenide glasses offer interesting prospects include Bragg solitons [76, 77] and photonic crystal cavities [78]. The mid-infrared transparency of these materials is also of significant interest and we expect increasing number of sensing devices for applications in molecular fingerprint analysis and astronomy.

\section{Acknowledgments}

This work was produced with the assistance of the Australian Research Council (ARC). CUDOS (the Centre for Ultrahigh-bandwidth Devices for Optical Systems) is an ARC Centre of Excellence. 